\title{
Advanced Equalization Techniques for Wireless Communications
}

\author{
Xiaoli Ma, ${ }^{1}$ Tim Davidson, ${ }^{2}$ Alex Gershman, ${ }^{3}$ Ananthram Swami, ${ }^{4}$ \\ and Cihan Tepedelenlioglu ${ }^{5}$ \\ ${ }^{1}$ School of Electrical and Computer Engineering, Georgia Institute of Technology, Atlanta, GA 30032, USA \\ ${ }^{2}$ Department of Electrical and Computer Engineering, McMaster University, Hamilton, ON, Canada L8S 4 K1 \\ ${ }^{3}$ Institute of Telecommunications, Communication Systems Group, Darmstadt University of Technology, 64283 Darmstadt, Germany \\ ${ }^{4}$ Army Research Laboratory, Adelphi, MD 20783, USA \\ ${ }^{5}$ Department of Electrical and Computer Engineering, Arizona State University, Tempe, AZ 85287, USA
}

Correspondence should be addressed to Xiaoli Ma, xiaoli@ece.gatech.edu

Received 31 December 2010; Accepted 31 December 2010

Copyright (C) 2010 Xiaoli Ma et al. This is an open access article distributed under the Creative Commons Attribution License, which permits unrestricted use, distribution, and reproduction in any medium, provided the original work is properly cited.

With the introduction of personal communications services, and digital packet data services, broadband wireless technology has experienced a significant upswing in recent years. To support the fast-growing wireless market, wireless systems must cope with the formidable challenges that stem from wireless fading and multipath effects, interference, finiteprecision DSP, high-signal dimension, and limited device size, to name a few. The goal is to design low-cost wireless devices that can communicate effectively at high data rates. To achieve this goal, an essential step is channel equalization. A desirable equalizer achieves high performance when operating at a high data rate and does so at low computational cost. The tradeoffs among performance, data rate, and complexity metrics are fundamental yet challenging in both the theoretical development and in hardware implementation. In this special issue, we have brought together state-of-theart research contributions that address advanced techniques for channel equalization in wireless communications.

Due to high-speed transmission and high-mobility terminals, equalization for time-varying and frequency-selective (a.k.a. doubly-selective) channels is an important issue. The paper by K. Fang et al. develops low-complexity receivers for orthogonal frequency-division multiplexing (OFDM) systems and single-carrier systems in doubly selective channels by embedding the channel estimation task within block turbo equalizers. Maximum likelihood semiblind joint channel estimation and equalization for doubly selective channels and single-carrier systems is proposed using expectationmaximization (EM) algorithm in the paper by G. Kutz and D. Raphaeli. The paper by C. Y. Yang and B.-S. Chen proposes a recursive maximum-likelihood (RML) algorithm for channel estimation under rapidly fading channel and colored noise in a multicarrier code-division multiple-access (MC-CDMA) system. The paper by F. Lehmann proposes blind equalization for block transmissions over frequencyselective Rayleigh fading channels, based on a Gaussian mixture parameterization of the a posteriori probability density function (pdf) of the transmitted data and the channel.

A significant portion of this special issue is devoted to channel estimation and equalization for multiple-input multiple-output (MIMO) systems. The paper by I. Barhumi and $\mathrm{M}$. Moonen proposes time-varying finite-impulse response equalization techniques for spatial multiplexingbased MIMO transmission over doubly selective channels. A blind bidirectional channel tracking algorithm, based on the projection approximation subspace tracking (PAST) algorithm, is applied to bidirectional time-varying MIMO channels in the paper by L. Ehrenberg et al. The paper by J. Tao et al. adopts a MIMO linear equalizer (LE) to remove space-time interference and a groupwise phase estimation and correction method to compensate the phase rotation in MIMO underwater acoustic (UWA) channels. J. Huang et al. describe a block-by-block iterative MIMO OFDM receiver with a channel estimator that exploits the sparsity of the UWA channel.

Certain channel characteristics and design criteria can be exploited to improve the performance of equalizers. The paper by H. C. Myburgh and J. C. Olivier proposes a softoutput low-complexity ML sequence estimation (MLSE) 
equalizer for channels with extremely long memory. The paper by D. Angelosante et al. casts the estimation of multiuser CDMA parameters as a sparse linear regression problem. For SIMO-OFDM transmissions with nonlinear clipping at the transmitter, the combination of equalization and mitigation of the nonlinearity has been considered in the paper by Q. Liu et al. The paper by L. Kovacs et al. proposes a novel equalization algorithm that is based on minimizing the Bit Error Rate (BER) using an efficiently computable approximation of its gradient. The paper by $\mathrm{M}$. Lipardi et al. derives optimum and suboptimum techniques for constellation design assuming a widely linear equalizer at the receiver.

There are two papers that consider equalization for ultrawideband (UWB) communications. The paper by Q. Zhou et al. proposes iterative multisymbol-based equalization methods that have low complexity and provide near optimal performance. S. Bahçeci and M. Koca consider the joint frequency-domain (FD) channel estimation and equalization for impulse radio UWB (IRUWB) systems with short cyclic prefix $(\mathrm{CP})$ and propose a novel iterative receiver employing soft interblock interference estimation and cancellation within both the FD channel estimator and FD equalizer components.

\author{
Xiaoli Ma \\ Tim Davidson \\ Alex Gershman \\ Ananthram Swami \\ Cihan Tepedelenlioglu
}

